\title{
FEM-BEM Numerical Procedure for Insertion Loss Assessment of an Engine Beauty Cover
}

\author{
E. Armentani ${ }^{1}$, R. Trapani ${ }^{1}$, R. Citarella ${ }^{*}, 2$ A. Parente ${ }^{3}$ and M. Pirelli ${ }^{3}$ \\ ${ }^{I}$ Department of Chemical, Materials and Production Engineering - University of Naples Federico II, Italy \\ ${ }^{2}$ Department of Industrial Engineering - University of Salerno, Italy \\ ${ }^{3}$ FIAT Group, Pomigliano d'Arco (NA), Italy
}

\begin{abstract}
In this paper a FEM-BEM numerical methodology to simulate soundproofing effects generated by an engine beauty cover was developed. Indeed, the engine beauty cover is not only an aesthetic element but also accomplishes the specific function of soundproofing and thus it is important to calculate the noise attenuation it can provide. The methodology was implemented by using the commercial software Virtual Lab, produced by LMS: the indirect BEM (IBEM), with a variational solution algorithm, was adopted to assess the acoustic scenario whereas the dynamic structural analysis was performed by the Finite Element Method (FEM). A correlation with experimental data obtained in the Fiat Research Centre in Pomigliano d'Arco (Naples) was carried out to verify the efficacy of such a method. The procedure can be applied to any structural element of the same typology (not only of automotive type), with the aim to numerically determine its effectiveness in noise attenuation. The numerical and experimental Insertion Loss showed a satisfactory degree of correlation in all the range of frequencies of relevant importance in the automotive field.
\end{abstract}

Keywords: Acoustic-vibration, engine beauty cover, insertion loss, noise absorption.

\section{INTRODUCTION}

It is common knowledge that the commercial success of a car directly depends on its quality parameters and their effect on passengers' and their safety on board. These parameters rely upon ergonomics, design, soundproofing as well as on many other factors that could influence the decisions of designers even in the early planning stages. Therefore, in order to achieve a stronger competitive market, it stands to reason that the quest for obtaining increasingly noiseless cars, has given rise to a considerable interest in studying sound transmission issues [1-3]. To this end, the simulation capabilities of the Virtual. Lab software, produced by LMS [4-10], proved to be very effective in obtaining higher soundproofing capacity of FIAT components.

In order to test the accuracy of the presented methodology, experimental data provided by the Fiat Research Centre of Pomigliano d'Arco (Naples) were compared with numerical outcomes.

The aforementioned virtual procedure, when calibrated, should allow to simulate the tentative design solutions for a passive [10] or active [11] reduction of the structural noise transmitted inside the car cabin, avoiding the need for expensive experimental tests.

\section{THEORETICAL BASIS OF BEM ACOUSTIC FORMULATION}

The indirect formulation [12] allow the contemporary resolution of the internal and external problem so it is

*Address correspondence to this author at the Department of Industrial Engineering - University of Salerno, Italy; Tel: +39089964111;

E-mail: rcitarella@unisa.it particularly useful when considering an open vibrating domain (like in our case). As a matter of fact, differently from the direct approach in which the problem variables are defined only on one side of the domain boundary, the indirect representation make usage of the layer potentials that are the differences between internal and external pressures (double layer potentials) or between the pressure gradients (single layer potentials).

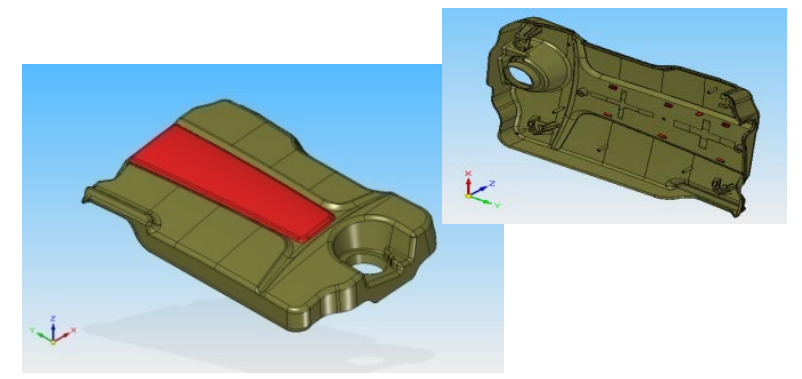

Fig. (1). Beauty cover engine.

The particle vibration velocity is calculated in a post processing phase as a function of the layer potentials.

In a well posed problem in each point of the surface one of the following boundary conditions must be specified:

- $\quad$ pressure,

- normal velocity,

- $\quad$ surface impedance, providing a relation between the two aforementioned variables.

The first step is then the calculation of unknown layer potentials through a solution scheme based on a variational formulation [12]. 
The variational scheme for the Neumann problem may be found, e.g., in Hamdi [13].

\section{COMPONENT TO BE ANALYSED: ENGINE BEAUTY COVER}

The analysed component consists of a beauty cover mounted on an engine cylinder head (Fig. 1). The cover represents not only an aesthetic component but it also ensures soundproofing capabilities, that are numerically and experimentally assessed in this paper.

The picture shown in Fig. (2) (realized by using a solid modelling software) gives a visual representation of the structural element position when assembled in the car.

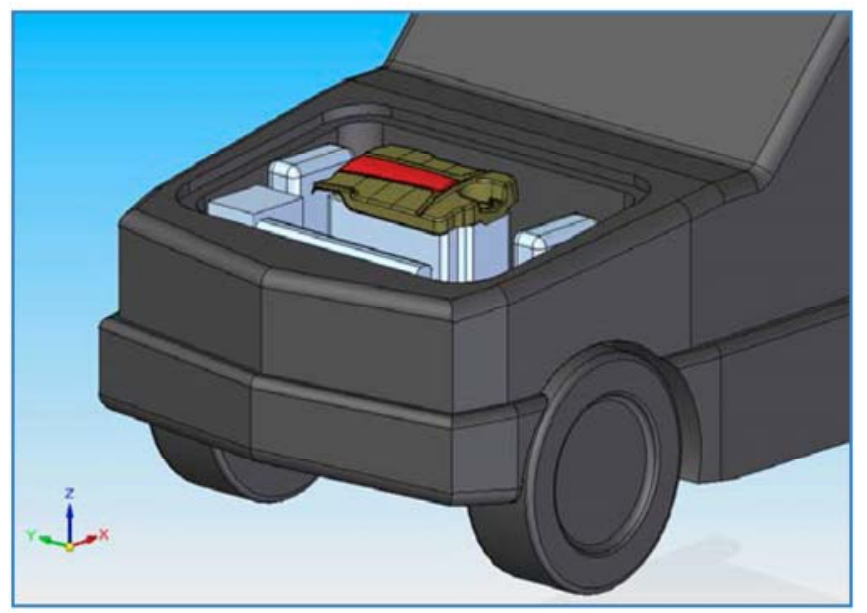

Fig. (2). Beauty cover engine position on car.

\section{ACQUISITION OF EXPERIMENTAL DATA}

The experimental data acquisition was carried out in the Fiat laboratories, precisely, in a semi-anechoic room for fit testing and included the following components:

- $\quad$ the engine beauty cover;

- a supporting framework for the beauty cover, stiffly anchored to the room reflecting floor (located 970 $\mathrm{mm}$ away from the cover itself);

- $\quad$ an acoustic source (LMS E-MHFVVS type), placed under the beauty cover, $25 \mathrm{~mm}$ away from its sides and $5 \mathrm{~mm}$ from a line normal to the beauty cover and passing through its centre;

- $\quad$ three free-field $1 / 2$ " omnidirectional microphones, respectively placed: one under the beauty cover and the other two above it, along a perpendicular line passing through the centre; the microphones placed above the cover are respectively $20 \mathrm{~mm}$ and $1000 \mathrm{~mm}$ from the cover, while the one below the cover is 20 $\mathrm{mm}$ from the cover itself;

- some supporting frameworks for both the microphones and the source;

- a set of instruments for the processing of data acquisition.

The measurements concerning those parameters were obtained through the LMS Test.LAB software [9].
With the aim of making clear the reciprocal positions of the components in the above mentioned framework, some representative schemes are shown in four groups:

- $\quad$ Supporting framework (Fig. 3);

- $\quad$ Position of the microphones A and B (Fig. 4);

- $\quad$ Position of microphone C and source (Fig. 5);

- Position of the beauty cover (Fig. 6).

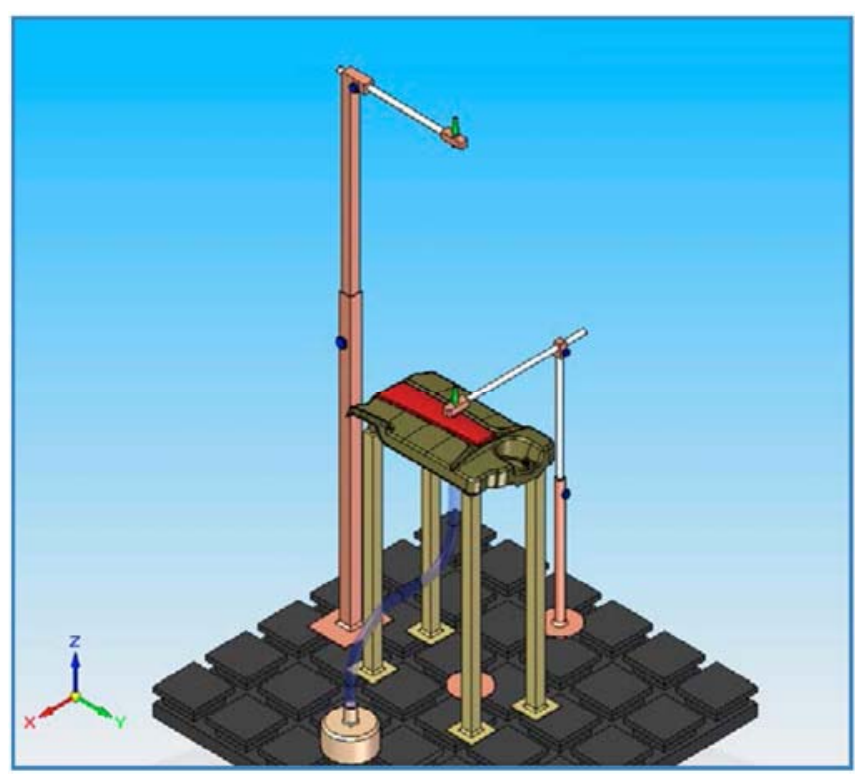

Fig. (3). Supporting framework.

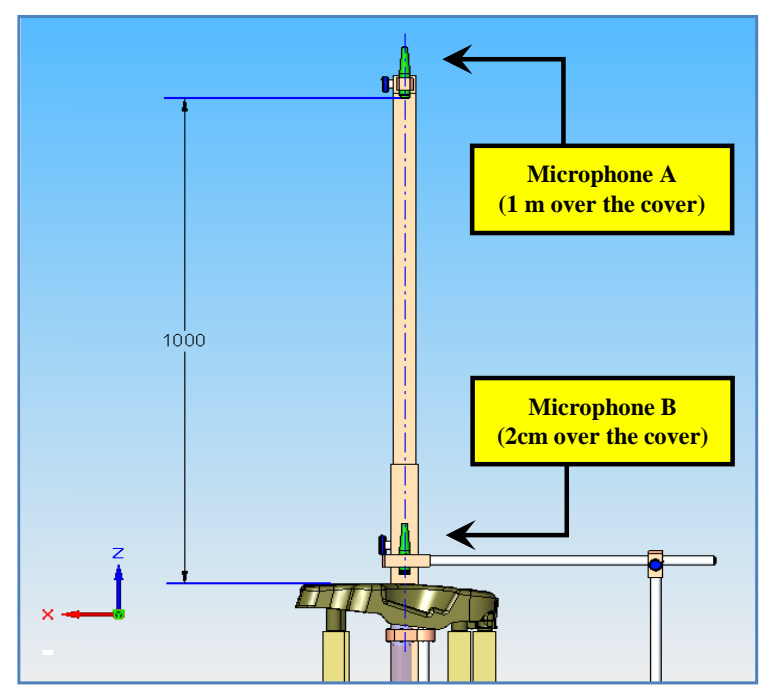

Fig. (4). Position of the microphones A and B.

The available experimental data are:

- amplitude of the sound pressure measured by microphone A;

- amplitude of the sound pressure measured by microphone B;

- amplitude of the sound pressure measured by microphone $\mathrm{C}$; 


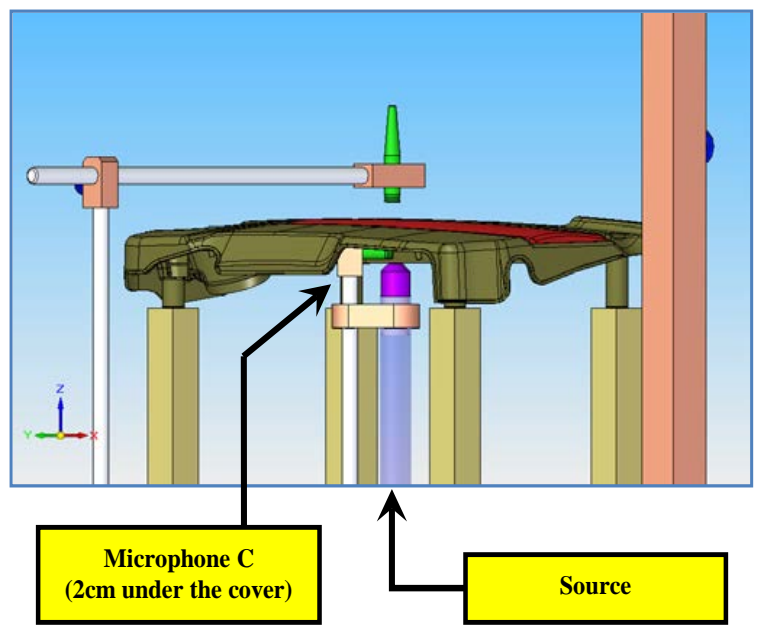

Fig. (5). Position of microphone $\mathrm{C}$ and source.

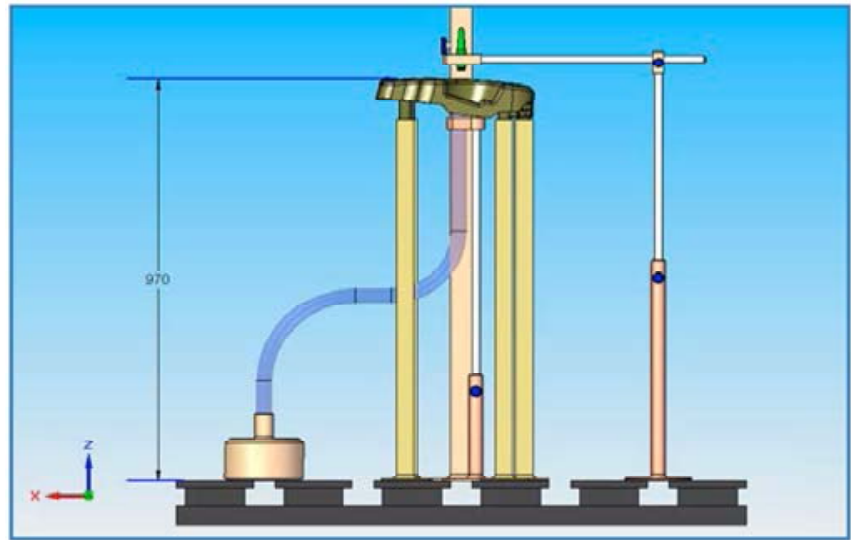

Fig. (6). Position of the beauty cover.

- $\quad$ amplitude and phase of the volumetric acceleration $\left(\mathrm{m}^{3} / \mathrm{s}^{2}\right)$ produced by the source.

All these parameters are frequency dependent and analysed in the range $200-800 \mathrm{~Hz}$.

\section{NUMERICAL MODEL}

The Virtual.Lab software was used to define the numerical model. The aim is to simulate the sound propagation attenuation, following the introduction of the engine beauty cover; obviously, being the extension of this component so restricted, the model must also take into account the sound waves propagating through the surrounding air.

The following sequence was carried out to define the numerical model for the beauty cover:

$>\quad$ creation of FEM model of the component and its modal analysis (Fig. 7);

$>\quad$ creation of an acoustic mesh (Fig. 8);

$>\quad$ creation of a structural file in Virtual.Lab;

$>\quad$ creation of an acoustic file in Virtual.Lab.

The beauty cover FEM model and the subsequent modal analysis were respectively realized by Hypermesh and Abaqus software. Several dynamic analyses were carried out considering a $3 \%$ structural damping (this value is considered appropriate for this structural component).

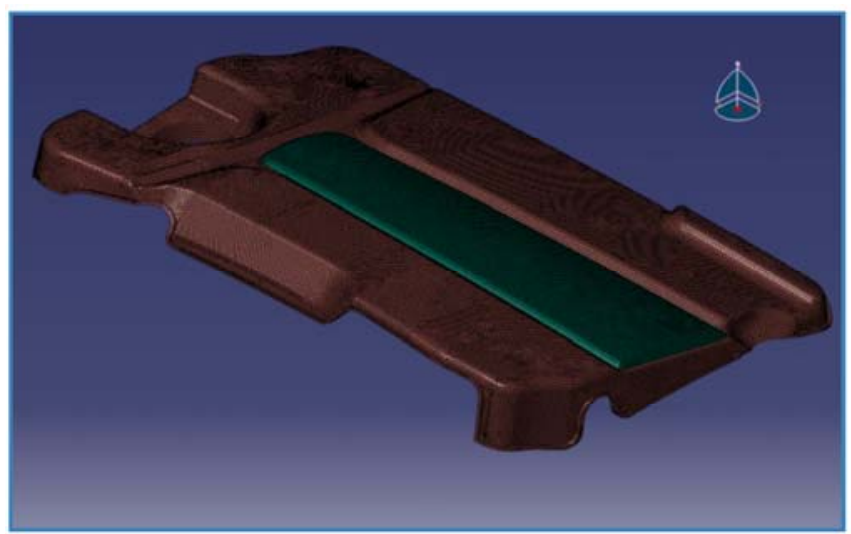

Fig. (7). Beauty cover FEM model.

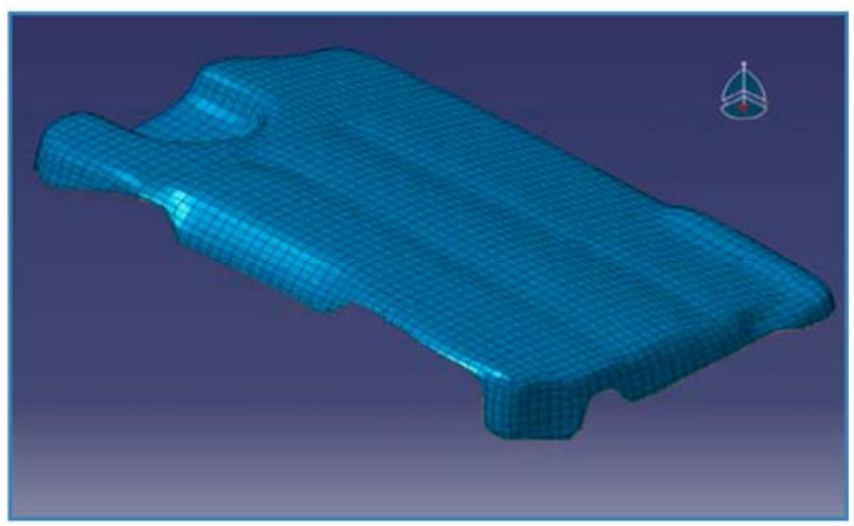

Fig. (8). Beauty cover acoustic mesh.

The FEM cover model was fully constrained in the violet highlighted zones shown in Fig. (9): these zones correspond to the contact points with the supporting framework of the experimental setup.

In order to simulate the source used during the experimental phase, the "source point" better known as "monopole" was adopted.

This choice stands for two main reasons:

1. omni-directionality of the experimental source: this property is properly fulfilled by the monopole because it is theoretically representative of a pulsating sphere of infinitesimal radius;

2. the emission of a signal from the effective source in volumetric acceleration $\dot{Q}\left[\mathrm{~m}^{3} / \mathrm{s}^{2}\right]$; this parameter is calculated from the relation:

$\dot{Q}=\frac{4 \pi A}{\rho_{o}}$

where $\rho_{o}$ is the air density $\left(1,225 \mathrm{~kg} / \mathrm{m}^{3}\right)$ and $A$ the polar sound amplitude emitted by the monopole.

Considering that the testing room is semi-anechoic, the simulation of this characteristic was achieved with a plane of symmetry placed at $970 \mathrm{~mm}$ from the cover (this plane simulates the room reflecting floor). The reciprocal positions of the virtual elements (microphones, source, plane of 
symmetry) included in the numerical model were defined with a similar procedure for the experimental fitting-up (Figs. 10-12).

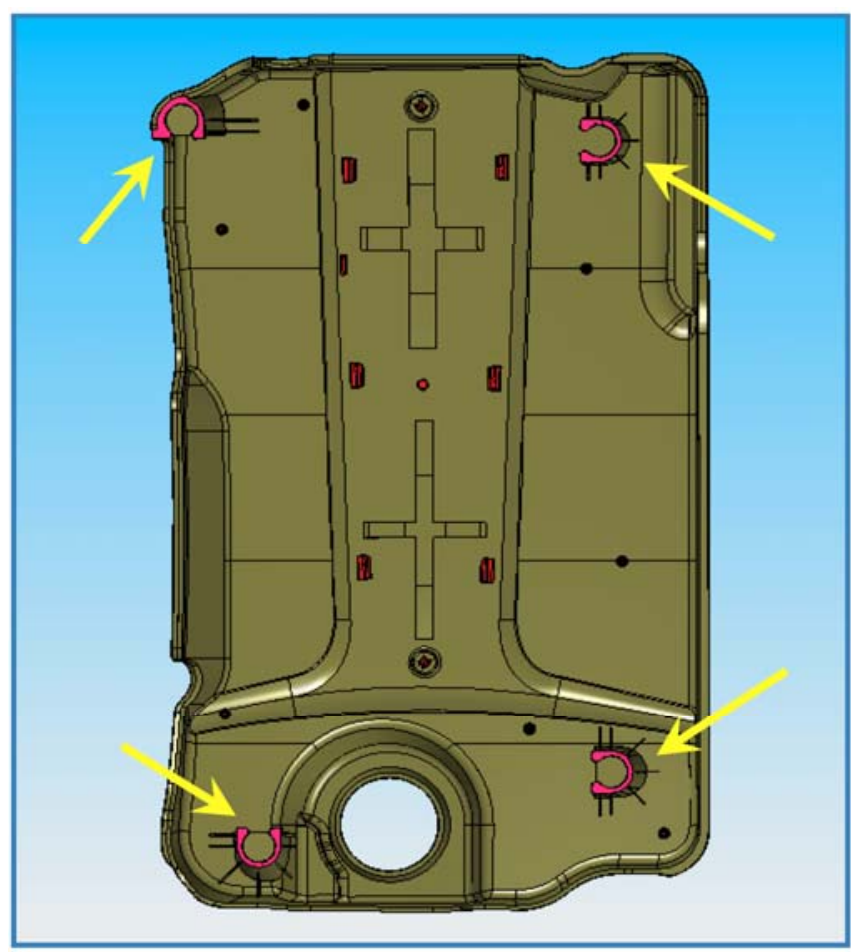

Fig. (9). Position of constraints.

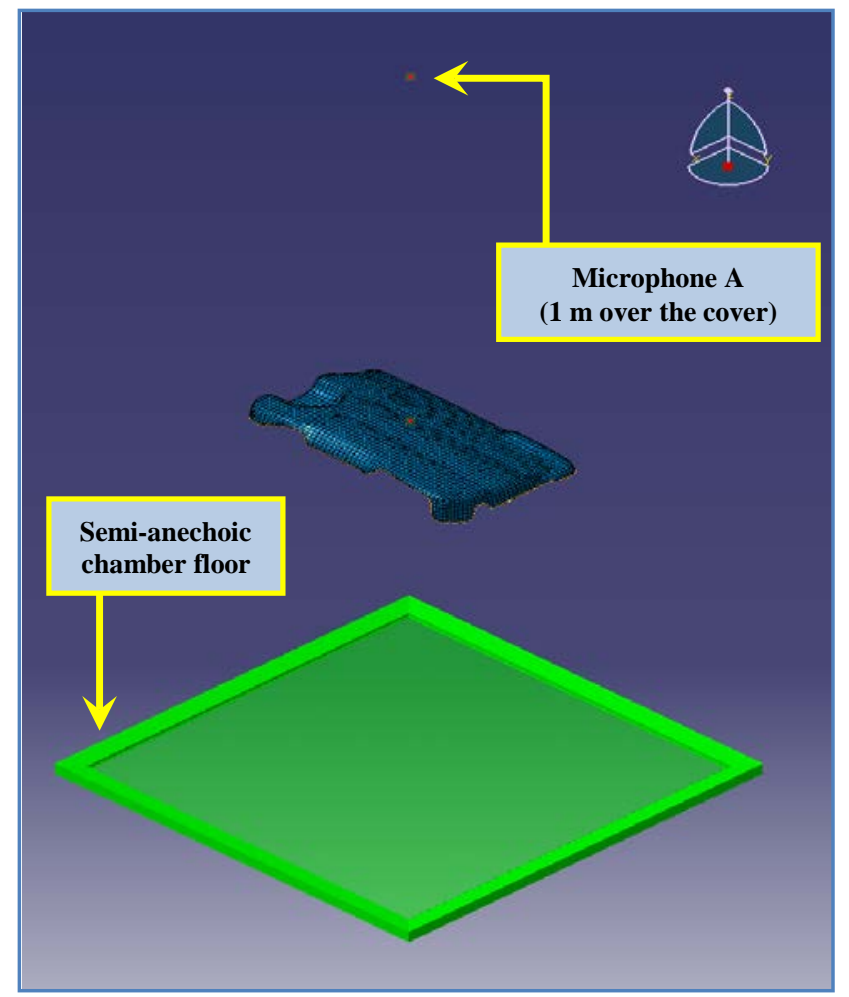

Fig. (10). Position of microphone A and plane of symmetry.

The following data were obtained from the elaborated numerical model:
- amplitude of the sound pressure detected by microphone A;

- amplitude of the sound pressure detected by microphone B;

- amplitude of the sound pressure detected by microphone $\mathrm{C}$.

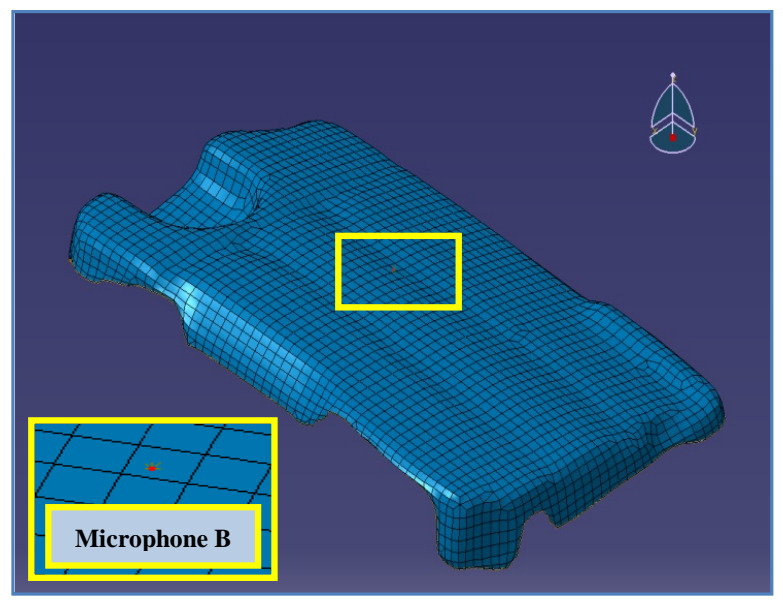

Fig. (11). Position of microphone B ( $2 \mathrm{~cm}$ above the cover).
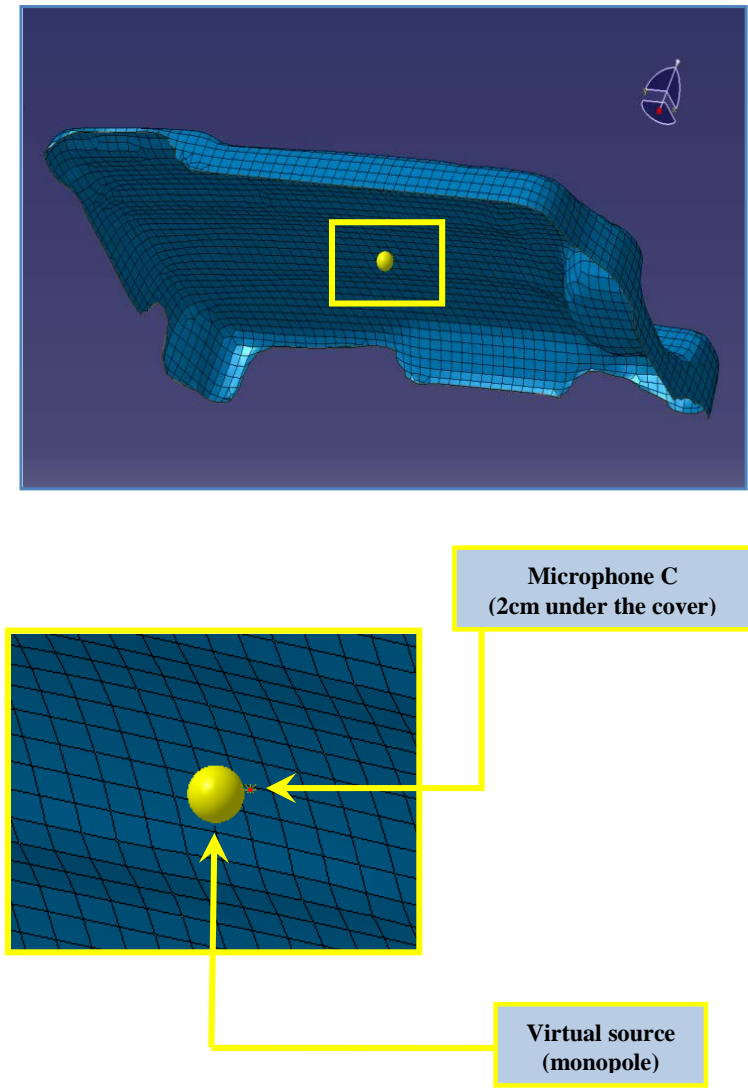

Fig. (12). Position of microphone $\mathrm{C}$ and source. 


\section{RESULTS AND DISCUSSION}

The level of correlation between numerical and experimental data is reported as follows:

* amplitude of the sound pressure detected by microphone A (1 m over the cover) (Fig. 13);

* pressure level detected by microphone A (1 m over the cover) (Fig. 14);

- amplitude of sound pressure detected by microphone B ( $2 \mathrm{~cm}$ over the cover) (Fig. 15);

* pressure level detected by microphone B $(2 \mathrm{~cm}$ over the cover) (Fig. 16);

- amplitude of sound pressure detected by microphone $\mathrm{C}$ ( $2 \mathrm{~cm}$ under the cover) (Fig. 17);

* $\quad$ pressure level detected by microphone C (2cm under the cover) (Fig. 18).

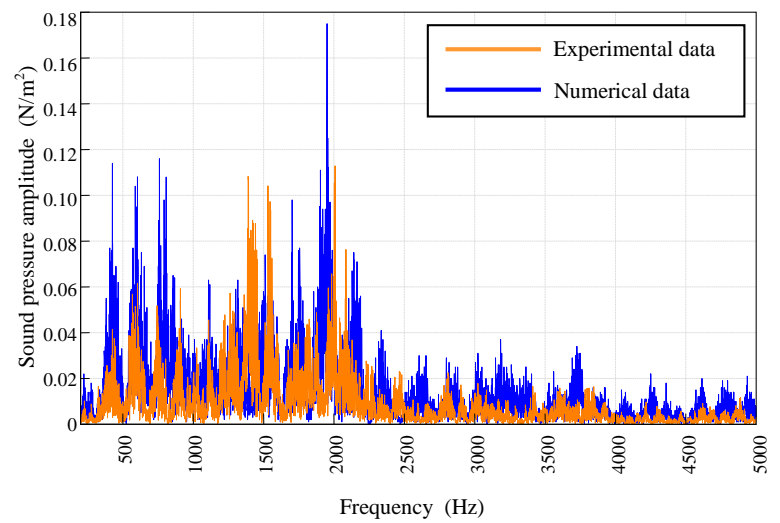

Fig. (13). Sound pressure amplitude - Microphone A.

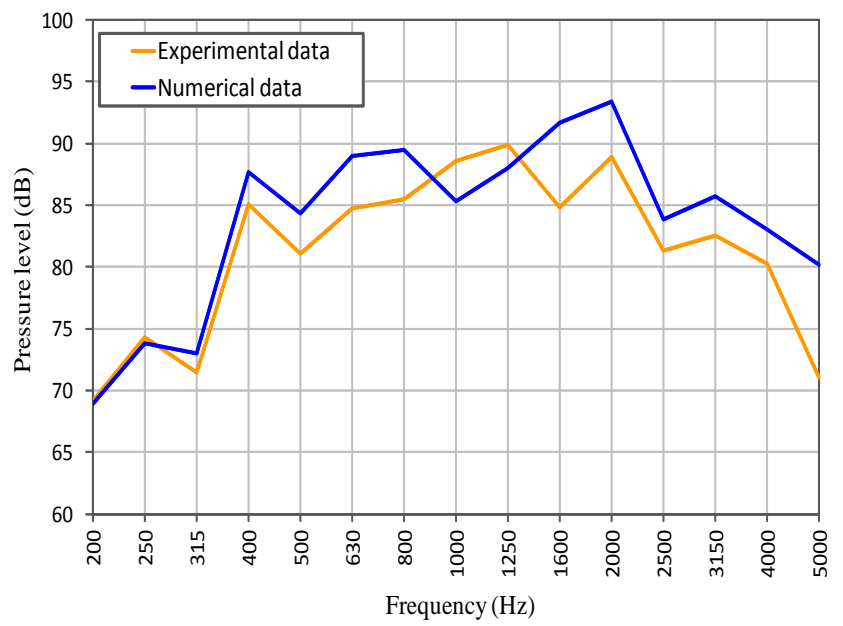

Fig. (14). Pressure level - Microphone A.

The reported diagrams show a satisfactory grade of correlation, which demonstrates the reliability of the realized numerical procedure.

In particular, it is possible to observe an almost systematic numerical over-esteem of the pressure levels; this can be due to a slight difference between the numerical and experimental microphone position.

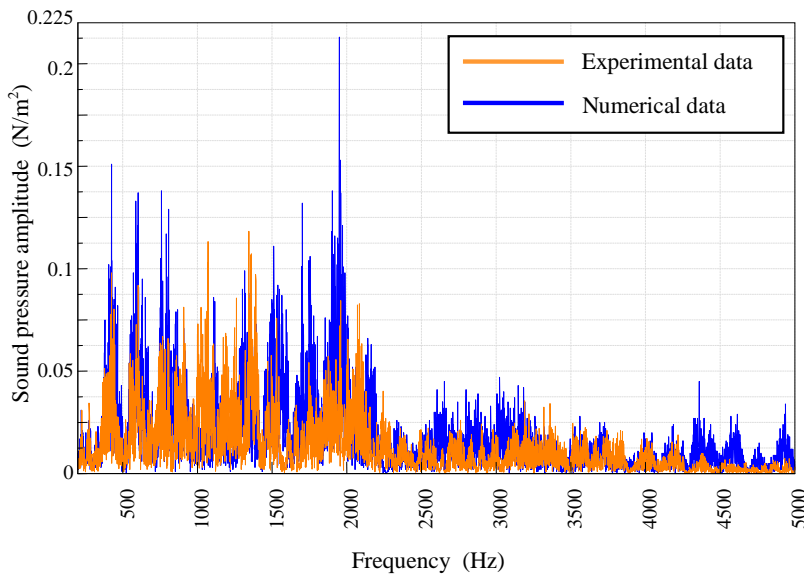

Fig. (15). Sound pressure amplitude - Microphone B.

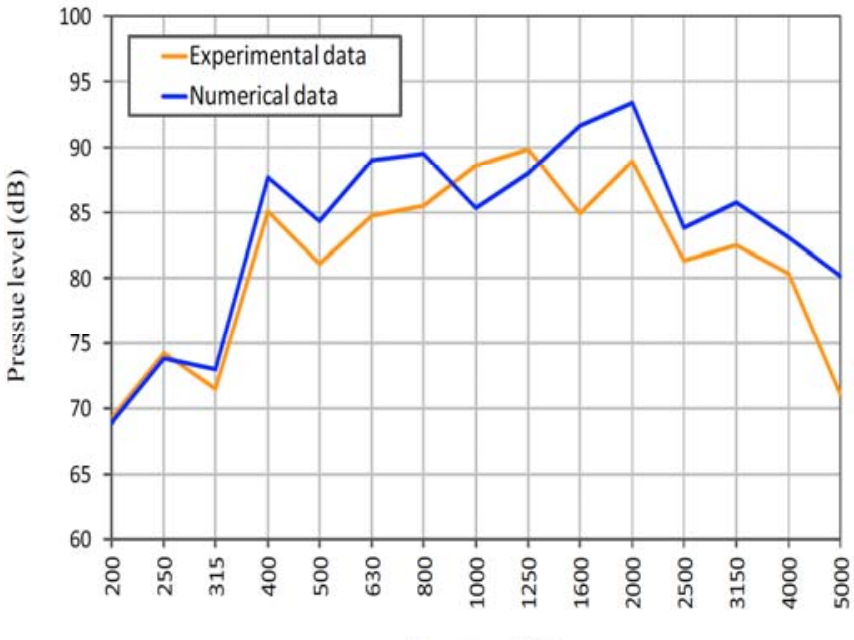

Frequency $(\mathrm{Hz})$

Fig. (16). Pressure level - Microphone B.

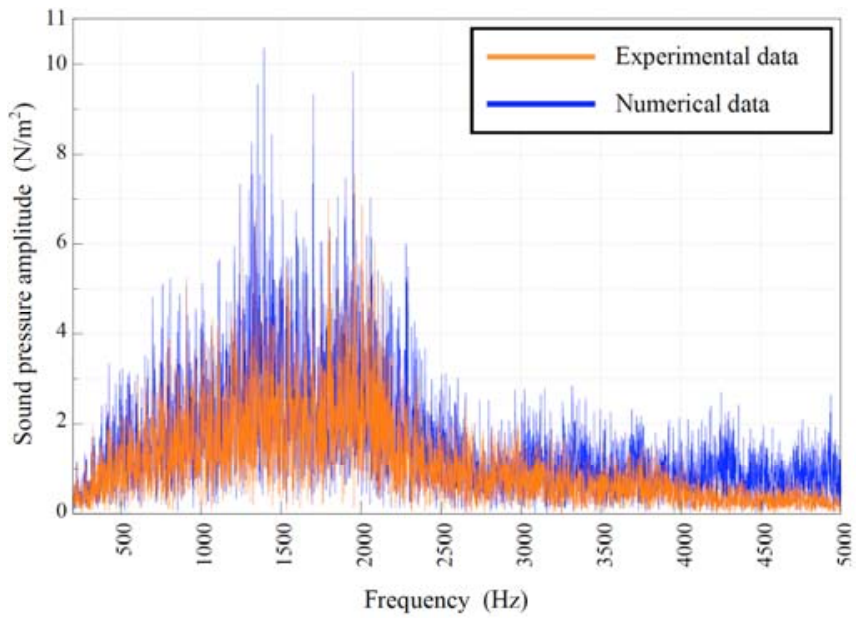

Fig. (17). Sound pressure amplitude - Microphone C.

A second phase of the numerical-experimental analysis provides the definition of a particular parameter capable to quantify the shielding action of the beauty cover with regard to sound transmission.

The shielding action of the beauty cover - in a given point - can be represented through the quantity Insertion Loss $I L$. This value is obtained from the difference between 
the levels of sound pressure in a determined position exposed to the noise, before and after the installation of the cover, with the same settings for the noise source and the same atmospheric conditions. This parameter is, then, expressed with the relation:

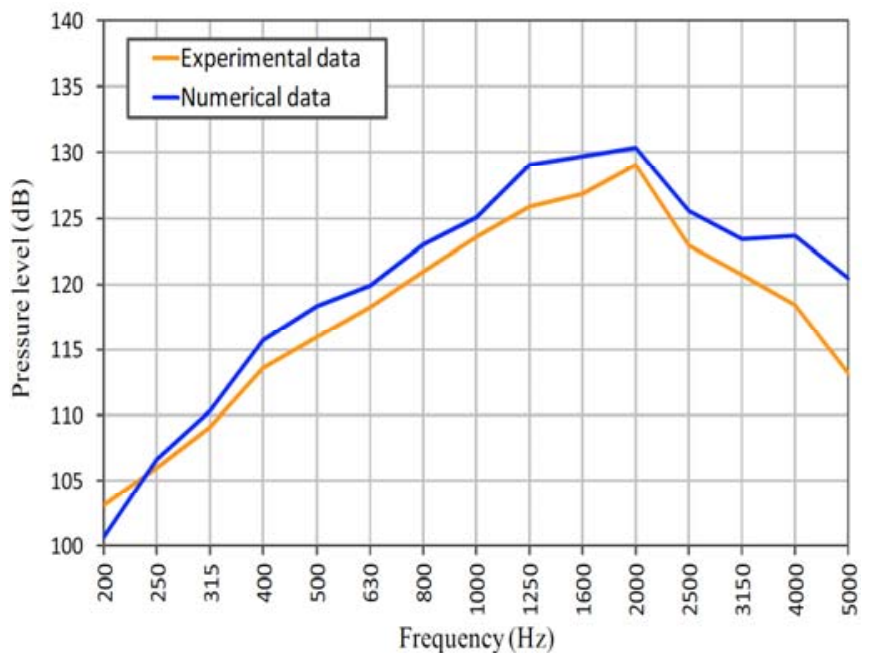

Fig. (18). Pressure level - Microphone C.

$$
I L=L_{p X,(\text { ante-operam })}-L_{p X,(\text { post-operam })} \quad[d B]
$$

where, $L_{p x \text { (ante-operam) }}$ indicates the sound pressure level before the insertion, while $L_{p x(\text { post-operam })}$ indicates the sound pressure level after the insertion of the shielding component.

In regard to the calculation of the Insertion Loss, the position of microphone A (1 m over the cover) was chosen as monitoring point for the noise attenuation. Consequently, the sound pressure level in that point was considered, with and without the cover. Moreover, it was deemed appropriate to report the corresponding diagrams in third of octave bands. The estimation of the Insertion Loss takes into account both the experimental data, obtained in semianechoic chamber, and the numerical data; the obtained diagrams were, then, compared in order to determine their degree of correlation.

The estimation of the Numerical Insertion Loss was carried out considering the numerically obtained data, therefore, the sound pressure level in A (1 m over the cover) was evaluated with and without the cover and then the above formula (2) was applied (Fig. 19).

The Experimental Insertion Loss was evaluated by taking into account the experimental data obtained in a semianechoic chamber; through these data the sound pressure level in A (1 m over the cover) can be determined, with and without the cover, and then the formula (2) of the Insertion Loss reported above was applied (Fig. 20).

Once extracted, the numerical and experimental diagrams, related to the Insertion Loss, were compared in order to determine their correlation degree (Fig. 21).

This comparison showed a satisfactory degree of correlation in all the analysed range of frequencies and, in particular, revealed to be optimum in the interval $2-5 \mathrm{kHz}$, interval of considerable importance in the automotive field.

\section{CONCLUSIONS}

Given the level of generality of the tested procedure, the model can be applied to any structural element of the same typology (not only of automotive type), with the aim to numerically determine its effectiveness in noise attenuation.

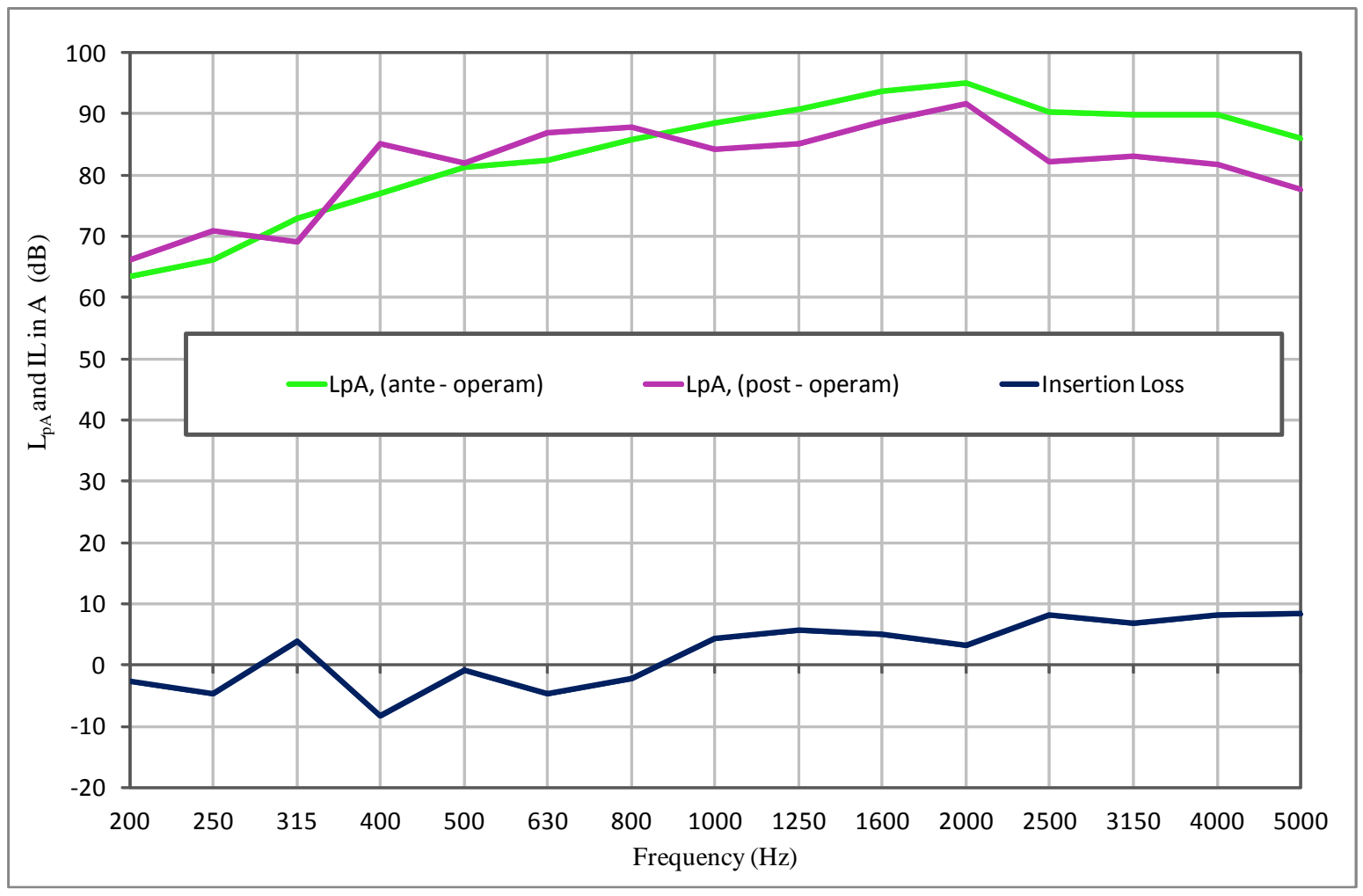

Fig. (19). Sound pressure levels in A (1 m over the cover) and Insertion Loss (numerical data). 


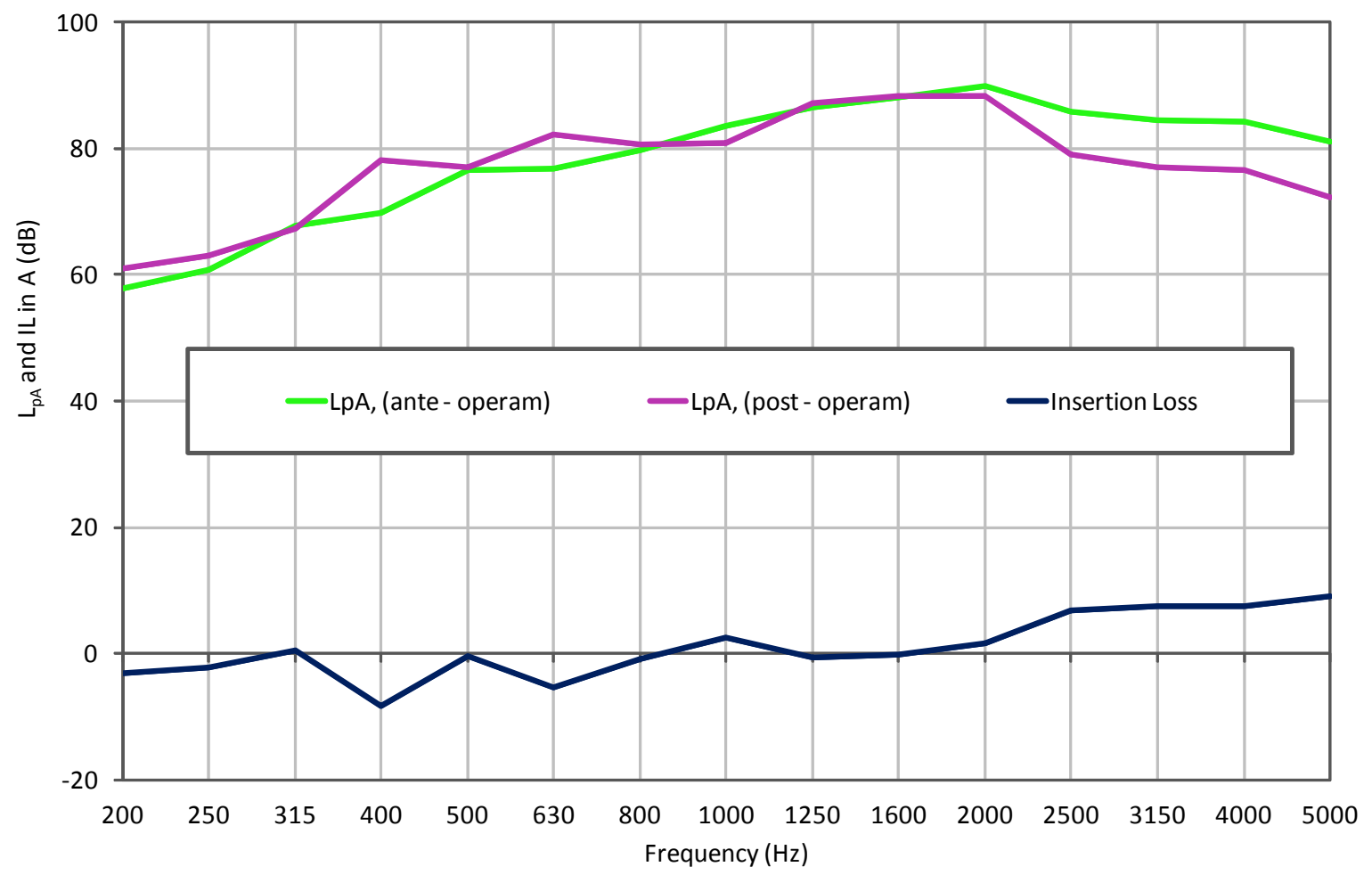

Fig. (20). Sound pressure levels in $A$ (1 $\mathrm{m}$ over the cover) and Insertion Loss (experimental data).

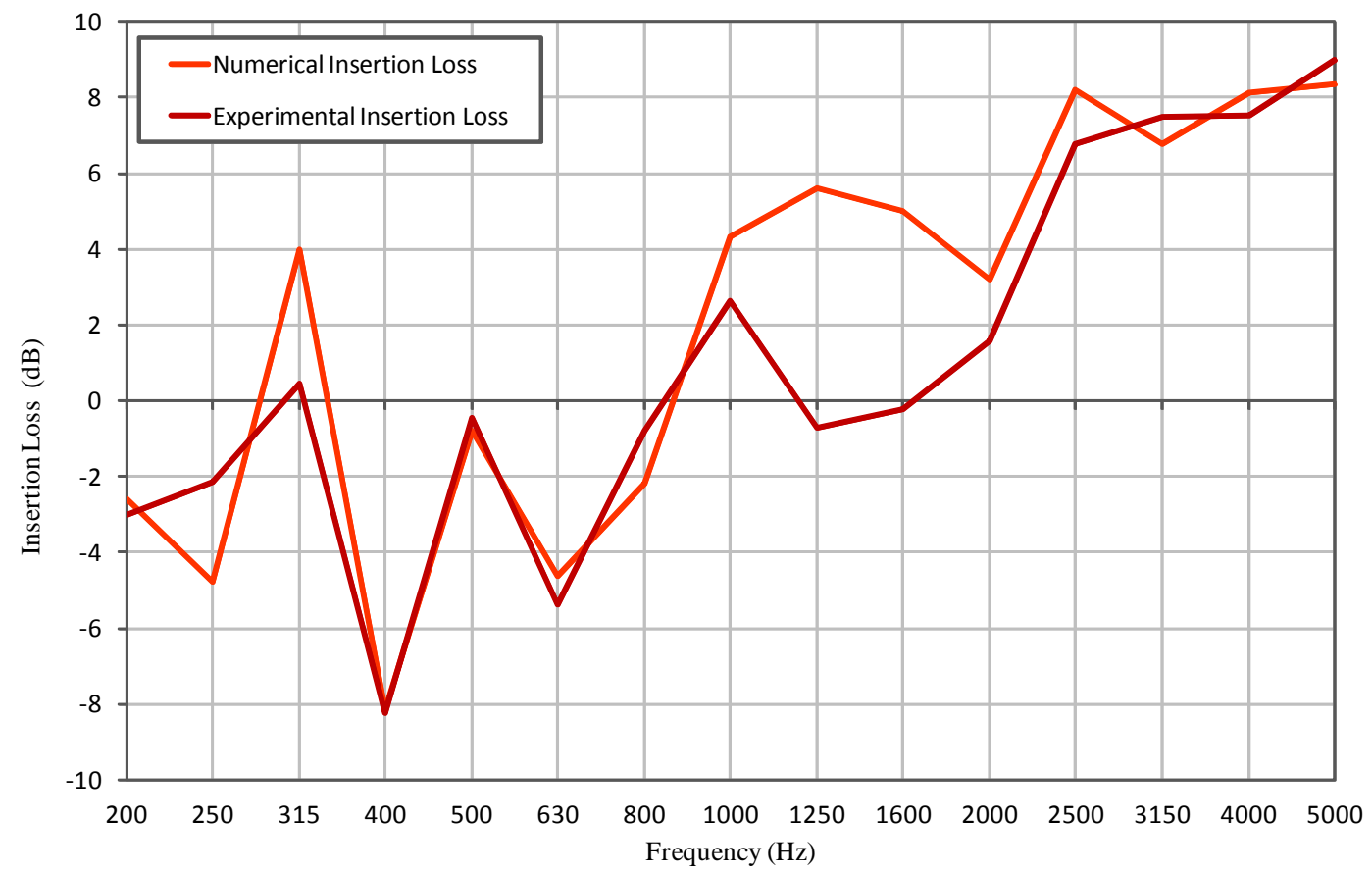

Fig. (21). Comparison between Insertion Loss in A (1 m over the cover).

Among the numerous advantages that the BE technology can offer in the acoustic field, are:

- $\quad$ a reduced discretisation effort;

- the possibility to easily evaluate the external problems in infinite domains; the possibility to easily characterise the surface acoustic behaviour by means of appropriate impedance values as boundary conditions;

and those offered by the an integrated FEM-BEM approach, like: 
- $\quad$ high flexibility;

- a potentially higher possibility of improving the numerical-experimental correlation (BEM is potentially more accurate than FEM).

In consequence of the aforementioned peculiarities it is possible to consider the FEM-BEM approach a valid alternative to the traditional FEM-FEM (both dynamic and acoustic analyses made by FEM) approach.

\section{CONFLICT OF INTEREST}

The authors confirm that this article content has no conflict of interest.

\section{ACKNOWLEDGEMENTS}

Declared none.

\section{REFERENCES}

R. Citarella, and D. Siano, "Transmission loss assessment of an air induction system by BEM", In: ICAD2006, The Fourth International Conference on Axiomatic Design, Firenze, Italy, June 2006, pp. 13-16.

[2] D. Siano, and E.F. Corcione, "FE fluid-structure interaction/ experimental transmission loss factor comparison of an exhaust system", In: 7th International Conference on Engines for Automobile - ICE2005, 2005.

[3] D. Siano, "Three-dimensional/one-dimensional numerical correlation study of a three-pass perforated tube", Simulation Modelling Practice and Theory, vol. 19, no. 4, pp. 1143-1153, 2011.
[4] R. Citarella, and M. Landi, "Acoustic analysis of an exhaust manifold by Indirect Boundary Element Method", The Open Mechanical Engineering Journal, vol. 5, pp. 138-151, 2011.

[5] R. Citarella, L. Federico, and A. Cicatiello, "Modal acoustic transfer vector approach in a FEM-BEM vibro-acoustic analysis, Engineering Analysis with Boundary Elements, vol. 31, pp. 248258. 2007.

[6] R. Citarella, P. Colantuono, and D. Siano, "Vibration prediction of a multi-cylinder engine using multi-body dynamic simulation", In: $41^{\circ}$ National Conference AIAS 2012, Vicenza: Italy, September 5$8,2012$.

[7] D. Siano, "Engine Noise Prediction by Using Multi-body Simulation", Journal of Environmental Science and Engineering B 1, pp. 1146-1161, 2012.

[8] D. Siano, and S. Giacobbe, "Radiated engine noise prediction using multi-body simulation", In: EURODYN2011, 8th International Conference on Structural Dynamics, Leuven: Belgium, 2011.

[9] LMS Virtual Lab, User's Guide.

[10] V. Mallardo, M.H. Aliabadi, A. Brancati, and V. Marant, "An accelerated BEM for simulation of noise control in the aircraft cabin", Aerospace Science and Technology, vol. 23, pp. 418-428, 2012.

[11] A. Brancati, M.H. Aliabadi, and V. Mallardo, "A BEM sensitivity formulation for three-dimensional active noise control", International Journal for Numerical Methods in Engineering, vol. 90, pp. 1183-1206, 2012.

[12] O. von Estorff, J.P. Coyette, and J.L. Migeot, Governing formulations of the BEM in acoustics", In: Boundary Elements in Acoustics - Advances and Applications, O. von Estorff, Ed., WIT Press: Southampton, 2000.

[13] M.A. Hamdi, "A variational formulation by integral equations for the solution of the Helmholtz equation with mixed boundary conditions" (in French), Compte-rendus Académie des Sciences, vol. 292, 1981. 